\title{
Spinal anaesthesia for laparoscopic cholecystectomy: A feasibility and safety study
}

\section{Gautam B}

Lecturer, Department of Anaesthesiology and Intensive Care, Kathmandu Medical College, Sinamangal, Nepal

\begin{abstract}
Background: Regional anaesthetic techniques have benefited those patients undergoing laparoscopic surgeries that are deemed high risk to receive general anaesthesia (GA). But spinal anaesthesia (SA) has not been routinely employed as the sole technique for laparoscopic cholecystectomy (LC).

Objective: This study was conducted to uncover feasibility and safety of SA for conducting LC.

Materials and methods: Twelve American Society of Anaesthesiologists' physical status I or II patients undergoing elective LC received SA using $4 \mathrm{ml}$ of $0.5 \%$ hyperbaric Bupivacaine mixed with $0.15 \mathrm{mg}$ Morphine. Peri-operative preparations and management were all standardised, with other drugs being only administered to manage anxiety, pain, nausea/vomiting, hypotension, and any adverse event. LC was performed with $\mathrm{CO}_{2}$ pneumoperitoneum maintained at an intra-abdominal pressure of less than $10 \mathrm{~mm} \mathrm{Hg}$ and with minimal operating table tilt. Peri-operative events, operative difficulty, hospital stay and patient satisfaction were studied.

Results: Spinal anaesthesia was adequate for surgery in all but one patient. Intraoperatively, two out of four patients who experienced right shoulder pain received Fentanyl. Two patients were given Midazolam for anxiety and one was given Ephedrine for hypotension. Operative difficulty scores were minimal and surgery in one patient was converted to open cholecystectomy. Postoperatively, pain scores were minimal and no patient demanded opioid. One patient required antiemetic for vomiting and one patient each suffered headache and urinary retention. 11 patients were discharged within 48 hours of surgery and patient satisfaction scores were very good.

Conclusion: Spinal anaesthesia with Morphine-mixed hyperbaric Bupivacaine is adequate and safe for elective LC in otherwise healthy patients and minimises postoperative pain and opioid use. Success and safety of this technique, however, necessitates knowledgeable patient, gentle surgical procedure, and co-operation among patient and members of the perioperative care team.
\end{abstract}

Key words: Hyperbaric Bupivacaine; intrathecal Morphine; laparoscopic cholecystectomy; spinal anaesthesia.

$\mathrm{E}$ ver since the laparoscopic approach established itself as the new "gold standard" for cholecystectomy", endotracheal GA is regarded as the gold standard anaesthetic technique for LC. This must have been based on the supposition that laparoscopy necessitates endotracheal intubation to prevent aspiration, abdominal and respiratory discomforts ${ }^{2,3}$ and hypercarbia, secondary to the raised intra-abdominal pressure with the induction of $\mathrm{CO}_{2}$ pneumoperitoneum, a defining step during LC.

Laparoscopic surgeries performed under combined epidural and GA have been demonstrated to be more efficient in controlling postoperative pain when compared to GA alone ${ }^{4}$. However, the use of epidural anaesthesia as a sole technique for performing LC has been reported only in patients considered unfit to receive GA, mainly those with severe respiratory disease ${ }^{2,5}$.
The first planned SA for conducting surgery in human was performed by August Bier in 1898, and since then it is ascribed to infrequent incidence of serious morbidities and mortalities compared to $\mathrm{GA}^{6,7}$; however, it has not been routinely used to conduct LC. Motamed, et $a l^{8}$ utilised SA as an adjunct to GA to effectively and safely prolong analgesia following LC. But, for the first time, SA was employed as a sole anaesthetic technique for conducting LC with nitrous oxide pneumoperitoneum by Hamad and El-Khattary9. Similarly, segmental thoracic SA was shown to be effective and safe for patients undergoing LC in a feasibility study ${ }^{10}$. Recently, Tzovaras $\mathrm{G}$ and colleagues, in a controlled randomised

Correspondence

Dr. Binod Gautam

Department of Anaesthesiology and Intensive Care

Kathmandu Medical College-Teaching Hospital

Sinamangal, Kathmandu, Nepal

E-mail: gautambinod@hotmail.com 
trial, have shown the feasibility of performing LC safely under SA in otherwise healthy patients and in addition, they concluded that SA results in remarkably minimal postoperative pain without limiting recovery ${ }^{11}$.

Encouraged by these conclusions, we designed to conduct a study to determine feasibility and safety of spinal anaesthesia employing hyperbaric Bupivacaine with low-dose Morphine for conducting elective LC with low pressure $\mathrm{CO}_{2}$ pneumoperitoneum and minimal operating table tilting in otherwise healthy patients.

\section{Materials and methods}

This study was conducted at Kathmandu Medical College-Teaching Hospital, Kathmandu, Nepal after the institutional ethics committee's approval. From September 1 to October 2009 all patients posted for LC who gave written informed consent for the procedure and study were considered eligible, provided that they fulfilled the following criteria: American Society of Anaesthesiologist's physical status I or II, between 18 and 65 years of age, and body mass index of $30 \mathrm{~kg}$ $\mathrm{m}^{2}$ or less. Exclusion criteria were acute cholecystitis, pancreatitis or cholangitis, suspected common bile duct stones, previous open upper abdominal surgery, patients receiving any other study drug and the presence of any condition complicating and contraindicating SA.

Patients were explained during the preoperative visit by the anaesthesiologist, that any anxiety, discomfort, or pain occurring during surgery would be dealt with intravenous medications or if they wished, conversion to GA. Additionally, they were made clear about scoring on the visual analogue scale (VAS; 0: no pain and 10: worst possible pain) and scoring of symptoms ( 0 , nil; 1 , mild; 2 , moderate; 3 , severe). Preoperative preparations were standardised for all patients. Each patient received, via the oral route, Diazepam $5 \mathrm{mg}$, Ranitidine $150 \mathrm{mg}$ and Metoclopramide $10 \mathrm{mg}$ on the night prior to surgery.

In the operating room, after establishing non-invasive monitoring, an intravenous access was achieved and $500 \mathrm{~mL}$ of Ringer Lactate solution was commenced. Ondansetron $4 \mathrm{mg}$ and Dexamethasone $8 \mathrm{mg}$ were administered intravenously in all patients. To prevent infection, patients were administered one dose of a third generation Cephalosporin. Pre-anaesthetic values of heart rate, mean arterial pressure, respiratory rate, and pulse oximetry were recorded.

SA was performed with the patient in the sitting position. With all aseptic precautions $2 \mathrm{ml}$ of $1 \%$ Lignocaine was used for infiltrating skin at the $\mathrm{L}_{2}-\mathrm{L}_{3}$ interspinous space. Through a 25 gauge introducer needle a 27 gauge pencil point spinal needle was introduced into the subarachnoid space at the $\mathrm{L}_{2}-\mathrm{L}_{3}$ intervertebral space using a midline approach. After confirming a free flow of cerebrospinal fluid, $4 \mathrm{~mL}$ of $0.5 \%$ hyperbaric Bupivacaine mixed with $0.15 \mathrm{mg}$ of Morphine was injected intrathecally within 20 seconds. Patients were then placed in the supine position and were made to stay in the Trendelenburg position till the level of sensory block as assessed with pin-prick stimulus every 30 seconds, confirmed to be at or above $\mathrm{T}_{6}$ level or for 3 minutes, whichever occurred earlier. Time taken from the patient's arrival inside operating room to the start of surgery, defined as the 'time for induction', was recorded.

LC was carried out employing same technical principles. Pneumoperitoneum was established by using the open laparoscopy technique with $\mathrm{CO}_{2}$ administration at a flow rate of $1 \mathrm{~L} / \mathrm{min}$ via the umbilical port and restricting intra-abdominal pressure at a maximum of $10 \mathrm{~mm} \mathrm{Hg}$. Immediately after the creation of pneumoperitoneum and prior to gall bladder dissection, $12 \mathrm{ml}$ each of $0.25 \%$ of Bupivacaine were instilled into the right subdiaphragmatic space and over the gall bladder. Head up and left lateral tilts of the operating table were employed as minimal as possible. Surgeons were prepared to ask for GA if they felt that the anaesthetic technique was adding to technical difficulty for the surgical procedure. An orogastric tube was inserted to decompress the stomach only if the surgeon demanded for it. Subhepatic drains were put depending upon the surgeons' preference.

Intraoperatively, patients were monitored throughout the procedure; clinical observation was made and haemodynamic status was recorded at five minute intervals. Hypotension, defined as mean arterial pressure decreasing by more than $20 \%$ below the preanaesthetic value, was treated with Ephedrine $6 \mathrm{mg}$ intravenous bolus and repeated every $3 \mathrm{~min}$ to titrate to the effect. Bradycardia, defined as a heart rate of or less than 60/min, was treated with $0.5 \mathrm{mg}$ Atropine, and hypertension was defined as a mean arterial pressure increasing by more than $20 \%$ above the pre-anaesthetic value. Patients were encouraged to report events such as any discomfort, abdominal or shoulder pain, headache, nausea and vomiting. Each of these events was scored and recorded. Anxiety was treated with Midazolam 2 mg and pain with Fentanyl 50 mcg intravenous boluses as required. Oxygen was administered at a flow rate of $5 \mathrm{~L} / \mathrm{min}$ via mask. Patients were allowed to observe the surgical procedure on a monitor screen if they wished to, and reminded of the possibility of conversion to GA if they felt any disappointment with SA.

Any conversion of anaesthetic or surgical technique was noted with specific reasons behind it. Durations of pneumoperitoneum as well as surgery were recorded. 
$\mathrm{CO}_{2}$ was evacuated carefully, as completely as possible, at the end of surgery. Time taken from the completion of surgery to the patient's transfer out of the operating room, defined as 'patient turn over time', was also recorded. Operating surgeons were requested to rank technical difficulty associated with the procedure on a 10 points scale ranging from 1 to $10(1$, no difficulty at all and 10, extremely difficult).

Postoperatively, all patients were provided with $3 \mathrm{~L}$ $\mathrm{min}^{-1}$ of Oxygen via nasal prongs and were infused 1 $\mathrm{L}$ of Ringer Lactate solution and $1 \mathrm{~L}$ of Dextrose $5 \%$ for the next 24 hours. Respiratory rate, heart rate, blood pressure and pulse oximetry values were recorded every hour. Respiratory depression was defined as a respiratory rate at or below 8 per minute and a pulse oximetry value of less than $90 \%$ at any given time in the postoperative period.

Postoperative analgesia was aimed to be provided with intravenous Paracetamol $300 \mathrm{mg}$ every 6 hours. Postoperative pain was assessed at both relaxed and stressed (sitting up position) conditions by using the visual analog scale at 1, 2, 4, 8, 12, 18, 24 and 48 hours after the completion of surgery. If the patient was not satisfied with postoperative pain control and had a VAS score of more than 3, intravenous Tramadol $50 \mathrm{mg}$ was utilised as a first rescue analgesia. If pain persisted 30 minutes after the Tramadol administration, Pethidine $25 \mathrm{mg}$ was supplemented intravenously as a second rescue analgesia, but only on patient's demand. Other events such as shoulder pain, nausea and vomiting, urinary retention, pruritus, and headache were also asked. If they occurred, these symptoms were scored and recorded. Patients were fed orally the morning after surgery and discharged 48 hours after surgery, unless complications had occurred.

Patients were asked at the time of discharge to rank their satisfaction with regards to the anaesthetic procedure on a score ranging from 0 to 10 . All patients were followed up 5 to 8 days later for removal of staplers at the port sites and were again requested to rate their overall postdischarge satisfaction on a similar scale.

\section{Results}

Out of 41 patients who appeared for laparoscopic cholecystectomy between September and October 2009, 22 eligible patients were approached of which 12
(Table 1) agreed to enter the study. Spinal anaesthesia was performed without difficulty in all patients and the highest level of sensory blockade achieved was at $\mathrm{T}_{4}$. SA was adequate for surgery in all patients except one who received GA.

Intraoperatively, four patients $(33.33 \%)$ experienced right shoulder pain and two of them received Fentanyl (Table 2). They responded well and surgery was accomplished without any further pain. Two patients required Midazolam for anxiety. Ephedrine was required in one patient, owing to her hypotension; although a mean of 1460 (SD 138) $\mathrm{ml}$ of crystalloid was infused intraoperatively. All other patients did not require any additional medication.

Surgical procedure in one patient was converted to open cholecystectomy and hepatico-jejunostomy for her choledocholithiasis which took 134 minutes and this patient required insertion of nasogastric tube and placement of sub-hepatic drain. In other patients surgery took an average of 42 (range 26-54) minutes and technical difficulty scores as ranked by the laparoscopist were minimal.

As presented in Table 4, abdominal pain assessed at 1, $2,4,8,12,18,24$ and 48 hours postoperatively were minimal and easily treatable. Postoperatively, four patients received analgesia with intravenous Tramadol and no patient demanded for Pethidine.

Postoperative events related to surgical and/or anaesthetic procedures are presented in Table 3. One patient suffered vomiting which was controlled with intravenous Ondansetron $4 \mathrm{mg}$. One patient developed typical post-dural puncture headache of mild severity. Some degree of shoulder pain persisted in two patients for up to 6 hours, which was mild and did not require additional analgesic. One patient required instant catheterisation for urinary retention.

Discharge from hospital at 48 hours after surgery was possible for 11 patients. One patient who underwent open cholecystectomy and hepatico-jejunostomy was discharged 16 days after surgery. There was no mortality and no major morbidity in any of the patients. At the time of discharge all patients reported overall satisfaction scores of 8 or above (out of 10). Postdischarge satisfaction scores were similar. 
Table 1: Patient details and outcome indicators. Data are mean (range), mean (SD) or number of patients. ASA, American Society of Anaesthesiologists' physical status

\begin{tabular}{|l|r|}
\hline Sex (M/F) (n) & $3 / 9$ \\
\hline Age (yr) & $32(21-48)$ \\
\hline BMI (kg m $\left.{ }^{-2}\right)$ & $26.6(24-30)$ \\
\hline ASA I: ASA II (n) & $9: 3$ \\
\hline Time for induction (min) & $11(9-16)$ \\
\hline Operative time (min) & $42(26-54)$ \\
\hline Pneumoperitoneum time (min) & $36(22-44)$ \\
\hline Intraoperative fluid (mL) & $1460(138)$ \\
\hline Conversion to general anaesthesia (n) & 1 \\
\hline Conversion to open surgery (n) & 1 \\
\hline Operative technical difficulty score & $2(1-4)$ \\
\hline Patient turn over time (min) & $3(1-5)$ \\
\hline Discharge from Hospital within 48 hrs (n) & 11 \\
\hline Patient satisfaction score at discharge (10:9:8) (n) & $2: 6: 4$ \\
\hline Post discharge patient satisfaction score (10:9:8) (n) & $4: 6: 2$ \\
\hline
\end{tabular}

Table 2: Intraoperative events

\begin{tabular}{|l|r|}
\hline Shoulder pain & 4 \\
\hline Hypotension & 1 \\
\hline Hypertension & 0 \\
\hline Bradycardia & 0 \\
\hline Abdominal discomfort/pain & 1 \\
\hline Fentanyl need & 2 \\
\hline Midazolam need & 2 \\
\hline
\end{tabular}

Table 3: Postoperative events, number of patients

\begin{tabular}{|l|r|}
\hline Nausea/vomiting & $2 / 1$ \\
\hline Right shoulder pain & 2 \\
\hline Urinary retention & 1 \\
\hline Post-dural puncture headache & 1 \\
\hline Respiratory depression & 0 \\
\hline Tramadol need & 4 \\
\hline Pethidine need & 0 \\
\hline
\end{tabular}

Table 4: Postoperative pain scores on Visual Analogue Scale

\begin{tabular}{|c|c|c|}
\hline Time (hr) & At rest & Sitting up \\
\hline 1 & $0(0-2)$ & $0(0-3)$ \\
\hline 2 & $0(0-3)$ & $0(0-3)$ \\
\hline 4 & $0(0-3)$ & $1(0-3)$ \\
\hline 8 & $1(0-3)$ & $1(0-4)$ \\
\hline 12 & $1(0-3)$ & $1(0-6)$ \\
\hline 18 & $2(0-4)$ & $2(0-7)$ \\
\hline 24 & $2(0-6)$ & $2(0-7)$ \\
\hline 48 & $2(0-7)$ & $2.5(0-6)$ \\
\hline
\end{tabular}




\section{Discussion}

Our study confirms that it is possible to carry out laparoscopic cholecystectomy safely under spinal anaesthesia with the patient breathing spontaneously; all it requires is more effort, gentleness and a readiness to supplement with intravenous adjuncts and if needed conversion to standard general anaesthesia. In addition, SA with low-dose Morphine-mixed hyperbaric Bupivacaineprovides prolonged postoperativeanalgesia. Pain scores in our patients were minimal throughout the hospital stay and no patient demanded for parenteral opioids. This could be attributed to a combination of several factors: maintained analgesia from the resolving sensory blockade from SA; pre-emptive instillation of intra-peritoneal Bupivacaine ${ }^{12,13}$; the avoidance of potential sore throat and stress response associated with endotracheal GA; minimal stress response associated with a minimal invasive anaesthetic procedure, like $\mathrm{SA}^{14,15}$; and the use of preoperative Dexamethasone ${ }^{13,16}$.

Adequate pain relief is obviously a pre-requisite for optimal recovery, early mobilisation and consequently the patients' comfort and satisfaction, the surgeons' favorite and the anaesthesiologists' optimism. Minimally invasive surgeries such as LC induce minimal tissue trauma and thus allow a significant reduction in postoperative pain and analgesic consumption compared to those after conventional open cholecystectomy ${ }^{17}$. Nevertheless, pain intensity after LC might be significant ${ }^{18,19}$; parietal pain, visceral pain and shoulder tip pain might adversely affect recovery and discharge times. This has been a serious concern, especially in the ambulatory surgery set up, and thus several agents and techniques have been proposed and evaluated ${ }^{8,12,13,18,19}$. As the genesis, time course, nature, and intensity of pain following LC is greatly variable, multimodal analgesia ${ }^{13,20,21}$ is far superior than relying just on primitive model of parenteral opioids. The concept of multimodal or balanced analgesia ${ }^{22}$ takes the advantage of the additive or synergistic effects by combining multiple agents or techniques with the concomitant reduction of side effects. Our study shows promising results for SA combined with multiregional local anaesthetic instillation in providing prolonged postoperative analgesia and a very good satisfaction in our carefully selected patients appearing for LC.

Anaesthetic technique in one patient in our study was converted to GA. This patient experienced severe abdominal discomfort after induction of pneumoperitoneum for which he received two boluses of $50 \mathrm{mcg}$ Fentanyl. Thereafter, he complained of mild pain during skin incision while making epigastric port; although, he had a sensory blockade level at $\mathrm{T}_{6}$. This patient was not comfortable with other two boluses of Fentanyl. We did not take any chances and decided to convert to GA. However if the epigastric port site was prepared a few centimeters downwards and had we applied pre-emptive local anaesthetic infiltration at the epigastric port site, this patient might have tolerated the procedure and SA might have sufficed.

Notable intraoperative event in our patients included right shoulder pain. Laparoscopy-related referred right shoulder pain, principally attributed to diaphragmatic irritation from $\mathrm{CO}_{2}$ pneumoperitoneum ${ }^{3,9}$ is a wellknown phenomenon ${ }^{23}$. Occasionally, this symptom could be intolerable for awake patients necessitating conversion to $\mathrm{GA}^{3}$. Four patients in our study experienced some degree of shoulder pain. Importantly, it was mild and tolerable in half of the cases and it did not necessitate conversion of anaesthetic technique. Two patients who reported severe shoulder pain received Fentanyl and remained calm afterwards. The incidence of intraoperative shoulder pain $(33.33 \%)$ in our study corresponds with the work done by van Zundert AAJ et $a l^{10}$ who reported $25 \%$ incidence of shoulder-tip pain during LC under SA. In contrast, Tzovaras G et $a l^{11}$ reported that $43 \%$ of patients who underwent LC under SA suffered intraoperative shoulder pain. Our figure contrasted with that from a report of laparoscopic surgery under epidural anaesthesia which showed $48 \%$ incidence of intraoperative shoulder pain ${ }^{5}$. It has to be noted though that the latter study had not utilised preemptive Bupivacaine instillation. The low incidence of shoulder pain in our patients could be attributed to our lower cutoff value of intra-abdominal pressure at 10 $\mathrm{mm} \mathrm{Hg}^{23}$ combined with the minimal tilting of operating table and in addition, the pre-emptive instillation of Bupivacaine into the right sub-diaphragmatic space and over the gall bladder ${ }^{12,13}$.

The incidence of shoulder pain following laparoscopic surgery under GA ranges between 30 and- $50 \%{ }^{23}$. Low incidence of postoperative shoulder pain in our study $(16.66 \%)$ which corresponds to a former study ${ }^{10}$ might relate to our patients who had been approached very carefully. Furthermore, lower intra-abdominal pressure $^{23}$, pre-emptive Bupivacaine installation ${ }^{12,13}$ at the right sub-diaphragmatic space and over the gall bladder, gentle liver retraction and minimal irrigation ${ }^{2}$ during the procedure and complete evacuation of residual $\mathrm{CO}_{2}$ at the end of surgery ${ }^{24}$ could have been contributory.

We chose a low-pressure pneumoperitoneum at a maximum of $10 \mathrm{~mm} \mathrm{Hg}$ of intra-abdominal pressure to minimise diaphragmatic irritation ${ }^{3,9,23}$ as well as abdominal and respiratory discomforts. The use of low-pressure pneumoperitoneum did not imperil the adequacy of surgical space and vision and subsequently 
all the procedures were completed with minimal technical difficulty. Obese patients (with a body mass index of more than $30 \mathrm{KG} / \mathrm{m}^{2}$ ) in whom a potentially higher intra-abdominal pressure is needed were excluded from our study to avoid probable technical difficulties. However, better muscular relaxation offered by $\mathrm{SA}^{5}$ might offset this problem enabling success of LC under SA in carefully selected obese patients, provided operating surgeons have an adequate experience and aptitude.

Although haemodynamic effects of $\mathrm{CO}_{2}$ pneumoperitoneum during SA have not been well studied, our experience didn't show any notable events apart from hypotension. Hypotension is a common undesired consequence of SA. One patient $(8.33 \%)$ in our study suffered from intraoperative hypotension. The low incidence of hypotension could be attributed to our liberal fluid therapy, low intra-abdominal pressure, minimal operating table tilts $^{25}$ and absence of any cardiovascular disease in our patients. Hypotension occurring in our patient was easily overcome with Ephedrine, and it did not essentially affect the planned procedure. It is unlikely that intrathecal Morphine administration would contribute to hypotension ${ }^{26,27}$. The increase in systemic vascular resistance occurring during laparoscopic surgeries has got multiple origins: a reflex sympathetic response to pneumoperitoneuminduced decline in cardiac output ${ }^{28}$; various neurohumoral factors ${ }^{29}$; or influence of posture ${ }^{30}$. The spinal sympathetic block may compensate for the increased sympathetic tone resulting from pneumoperitoneum in a head-up position and offsets the vasomotor constriction of splanchnic organs and leg muscles. This might explain why none of our patients experienced hypertension intraoperatively. Although, SA is said to facilitate unopposed vagal reflex, none of our patients suffered bradycardia. Vasodilation due to SA-induced sympathetic blockade and avoidance of positive pressure ventilation might probably lessen the haemodynamic perturbations attributed to $\mathrm{CO}_{2}$ pneumoperitoneum during laparoscopic surgeries.

Altogether three patients reported nausea postoperatively. One of them experienced 3 episodes of vomiting six hours after surgery before being treated with Ondansetron: whereas the other two had mild nausea requiring no treatment. Without prophylaxis, up to $50-70 \%$ of patients following LC under GA have been reported to suffer from postoperative nausea and vomiting ${ }^{31,32}$. Low need for rescue antiemetic in our patients $(1 / 12)$ could be attributed to: antiemetic prophylaxis with Ondansetron and Dexamethasone ${ }^{16,33,34}$, avoidance of hypotension, adequate pain control and avoidance of postoperative opioid use. Postoperative urinary retention developed in 1 patient; this is known to be related to spinal anaesthesia ${ }^{35}$. The incidence of urinary retention after surgery may be further increased in patients receiving intrathecal Morphine ${ }^{26,27}$. Instant catheterisation was required with no adverse effect on the patient's recovery as well as discharge times.

At the time of discharge, all patients reported being highly satisfied with the anaesthetic approach which could be attributed to a good postoperative pain control, minimal nausea and vomiting, less fatigue and a good feeling of well being ${ }^{13,16}$. In addition, these patients were constantly educated and reassured perioperatively; patient motivational issues may also have been implicated as most of the patients had chosen to be awake during their surgery. Adequate explanation to the patient regarding possible requirements of intravenous analgesics and anxiolytics, or conversion to general anaesthetic technique is vital for the success of SA. Preoperative patient information regarding the perioperative care plan aids coping, reduces anxiety and enhances recovery; in addition, the knowledgeable patient requires less analgesia postoperatively ${ }^{36}$.

However, performing $\mathrm{LC}$ at a low intra-abdominal pressure and minimal operating table tilt might pose technical difficulties for the operating surgeons; but with increasing expertise, it is likely that one day surgeons might not hesitate to acknowledge SA as an effective technique for LC. Another possible problem in an awake, possibly anxious and hyperventilating patient is an inadvertent injury to the intra-abdominal structures. No patient in our study suffered inadvertent injury to the visceral organs. This was made possible by the surgeons being gentle and tuned with the patient's respiratory movements. In addition, co-operative patients were asked to withhold their breath during clipping and cutting of the cystic artery and cystic duct.

The first apprehension associated with SA with blockade extending up to $\mathrm{T}_{4-5}$ level is a possible ventilatory impairment. Although forceful expiration may get affected because it primarily entails anterior abdominal wall muscles which are innervated by thoracic nerves, the main inspiratory muscle namely diaphragm will be spared as its supply, the phrenic nerve, originates from the cervical level ${ }^{37}$. No patient in our study had pre-existing respiratory disease and no one experienced dyspnoea during abdominal insufflations, perhaps because of the low intra-abdominal pressure, and the highest sensory blockade achieved being $\mathrm{T}_{4}$. The anxiety associated with SA with hyperbaric Bupivacaine and employing head down (10 degrees) tilt for 3 minutes posing a risk of total spinal blockade is unfounded; as the highest level of sensory blockade attained in our patients was at $\mathrm{T}_{4}$. 
A next concern is the associated adverse effects of intrathecal Morphine including respiratory depression, vomiting, urinary retention and itching ${ }^{26,27,38}$. Once injected intrathecally, hydrophilic opioids like Morphine tend to remain within the cerebrospinal fluid and produce a delayed but a longer duration of analgesia ${ }^{39}$ along with a higher incidence of side effects due to their cephalad spread ${ }^{26,38}$. But in our set up, where patients are monitored on an inpatient basis after LC, a single lowdose of intrathecal Morphine administration proved to be safe as no patient in our study suffered respiratory depression. As the most dreaded adverse effects of intrathecal Morphine including respiratory depression and vomiting are dose dependent ${ }^{26,38}$, avoiding more than a recommended dose $\mathrm{e}^{8,38,40}$, efficient patient monitoring and preparedness to promptly deal with any adversity are the key to formulate intrathecal Morphine a safe and beneficial practice.

Lower frequency of serious Peri-operative morbidities and an improved outcome are often ascribed to SA compared to $\mathrm{GA}^{6,7}$; this is why regional anaesthesia is preferred for sicker patients. It's true that SA may not be the first choice for many operations; even though, today, GA as the only appropriate technique for LC must be a concept of history. Although this was not a comparative study, the spinal anaesthetic technique apparently increased time for anaesthetic induction (11 minutes). But this additional performance time investment was rewarded with smooth recovery, rapid patient turn over times (3 minutes), prolonged postoperative analgesia and high patient satisfaction scores.

Obviously, researchers tend to seek for techniques associated with minimal morbidity and expense for patients; and application of any newer approach to Perioperative care becomes a reality only after evaluating its feasibility and safety. It has been stated that the decisions and actions made by anaesthesiologist, as a key Peri-operative physician, are of critical importance to the surgical care team in developing a successful fasttrack surgery program ${ }^{41}$. Till date, LC is not performed on an ambulatory basis in our institution but in the future, with adequate experience, we might achieve a change in that trend. Socioeconomic pressures from limited healthcare resources in a country like ours have long been motivating us for evaluating techniques that might minimise the overall medical costs without compromising surgical outcome. Today, there is a growing body of evidence suggesting that regional anaesthesia has an important role to play in this regard. Utilising the well known assets of SA, like reduced metabolic response and better muscular relaxation together with a reduced need for sedatives and opioids, and adding to it the prolonged analgesic effects of low- dose Morphine proves to allow a favourable risk-benefit ratio. However, the scope of SA ultimately depends on the co-operation and creativeness of the Peri-operative care team.

With improvements in surgical technique and advances in Peri-operative anaesthetic care to achieve a painand risk- free course, aimed at shortening convalescent recovery, reducing the use of hospital resources and increasing patient satisfaction continue to remain our major concern. This doesn't seem to be beyond our reach provided we acknowledge a culture of accepting challenges as the real dominant aspects of anaesthesia practice are known to involve dynamism, time pressure, intensity, complexity, uncertainty and risk $^{42}$. But, internal or external pressure on the anaesthesiologist to keep more than one operating room schedule move along speedily, with few cancellations and minimum time between cases, known as production pressure, might be an important reason for an anaesthesia personnel being reluctant to employ newer techniques. However, anaesthesia continues to be a unique speciality that demands of its practitioners something approaching perfection; and there are always areas for potential improvement.

\section{Conclusion}

In conclusion, we believe that this is the first study to provide an indication regarding adequacy and safety of lumbar spinal anaesthesia with hyperbaric Bupivacaine and Morphine for conducting elective laparoscopic cholecystectomy in otherwise healthy patients. Our study has confirmed the feasibility of performing elective laparoscopic cholecystectomy with low pressure $\mathrm{CO}_{2}$ pneumoperitoneum and minimal operating table tilt under sole spinal anaesthesia. Additionally, it appears that spinal anaesthesia provides minimal intraoperative haemodynamic perturbations and is valuable in postoperative pain control and patient satisfaction. However, this approach requires a co-operative patient, an experienced laparoscopist amply skilled in precise and gentle surgical technique and an enthusiastic anaesthesiologist ever prepared to supplement it with intravenous adjuncts and if needed to convert the anaesthetic technique to general anaesthesia. From these conclusions, it becomes clear that with proper application and with suitable improvements, spinal anaesthesia has got the potential to emerge as the novel gold standard anaesthetic technique for elective laparoscopic cholecystectomy. A further randomised controlled trial is underway to compare spinal anaesthesia and general anaesthesia in terms of cost, benefits and risks while conducting elective laparoscopic cholecystectomy. 


\section{References}

1. Soper NJ, Stockman PT, Dunnegan DL, et al. Laparoscopic cholecystectomy: the new "gold standard"? Arch Surg. 1992; 127:917-21.

2. Pursnani KG, Bazza Y, Calleja $M$, et al. Laparoscopic cholecystectomy under epidural anesthesia in patients with chronic respiratory disease. Surg Endosc. 1998; 12(8): 1082-4.

3. Sharp JR, Pierson WP, Brady CE. Comparison of $\mathrm{CO}_{2}$ and $\mathrm{N}_{2} \mathrm{O}$-induced discomfort during peritoneoscopy under local anesthesia. Gastroenterology. 1982; 82(3): 453-6.

4. Luchetti M, Palomba R, Sica G, et al. Effectiveness and safety of combined epidural and general anesthesia for laparoscopic surgery. Reg Anesth. 1996; 21(5): 465-9.

5. Gramatica L Jr, Brasesco OE, Luna MA, et al. Laparoscopic cholecystectomy performed under regional anesthesia in patients with obstructive pulmonary disease. Surg Endosc. 2002; 16(3): 472-5.

6. Rodgers A, Walker N, Schug S, et al. Reduction of postoperative morbidity and mortality with epidural or spinal anesthesia: results from overview of randomized trials. Br Med J. 2000; 321: 1493-508.

7. Kopp SL, Horlocker TT, Warner ME, et al. Cardiac arrest during neuraxial anesthesia: frequency and predisposing factors associated with survival. Anesth Analg. 2005; 100: 85565.

8. Motamed C, Bouaziz H, Franco D, et al. Analgesic effect of low-dose intrathecal morphine and bupivacaine in laparoscopic cholecystectomy. Anaesthesia. 2000; 55(2): 118-24.

9. Hamad MA, Ibrahim El-Khattary OA. Laparoscopic cholecystectomy under spinal anesthesiawithnitrousoxidepneumoperitoneum: a feasibility study. Surg Endosc. 2003; 17(9): 1426-8.

10. van Zundert AAJ, Stultiens G, Jakimowicz JJ, et al. Laparoscopic cholecystectomy under segmental thoracic spinal anaesthesia: a feasibility study. Br J Anaesth. 2007; 98: 6826.

11. Tzovaras G, Fafoulakis F, Pratsas K, et al. Spinal vs general anesthesia for laparoscopic cholecystectomy. Arch Surg. 2008; 143(5): 497-501.

12. Boddy AP, Mehta S, Rhodes M. The effect of intraperitoneal local anesthesia in laparoscopic cholecystectomy: a systematic review and metaanalysis. Anesth Analg. 2006; 103: 682-8.
13. Bisgaard T. Analgesic treatment after laparoscopic cholecystectomy; a critical assessment of the evidence. Anesthesiology. 2006; 104(4): 835-46.

14. Aono H, Takeda A, Tarver SD, et al. Stress responses in three different anesthetic techniques for carbon dioxide laparoscopic cholecystectomy. J Clin Anesth. 1998; 10(7): 546-50.

15. Kehlet $H$. The stress response to surgery: Release mechanisms and the modifying effect of pain relief. Acta Chir Scand Suppl. 1998; 550: 22.

16. Bisgaard T, Klarskov B, Kehlet H, Rosenberg J. Preoperative dexamethsone improves surgical outcome after laparoscopic cholecystectomy. A randomized double-blind placebo-controlled trial. Ann Surg. 2003; 238: 651-60.

17. Joris J, Cigarini I, Legrand M, et al: Metabolic and respiratory changes after cholecystectomy performed via laparotomy or laparoscopy. Br J Anaesth. 1992; 69: 341.

18. Ure BM, Troidl H, Spangenberger W, et al: Pain after laparoscopic cholecystectomy; Intensity and localization of pain and analysis of predictors in preoperative symptoms and intraoperative events. Surg Endosc. 1994; 8: 90.

19. Joris J, Thiry E, Paris P, et al: Pain after laparoscopic cholecystectomy; characteristics and effect of intrapertoneal bupivacaine. Anesth Analg. 1995; 81: 379.

20. Michaloliakou C, Chung F, Sharma S. Preoperative multimodal analgesia facilitates recovery after ambulatory laparoscopic cholecystectomy. Anesth Analg. 1996; 82: 44.

21. Bisgaard T, Klarskov B, Kristiansen VB, et al. Multiregional local anesthetic infiltration during laparoscopic cholecystectomy in patients receiving prophylactic multimodal analgesia: A randomized, double-blinded, placebo-controlled study. Anesth Analg. 1999; 89: 1017.

22. Kehlet H, Werner M, Perkins F. Balanced analgesia. What is it and what are its advantages in postoperative pain? Drugs. 1999; 58: 793807.

23. Sarli L, Costi R, Sansebastiano G, et al. Prospective randomized trial of low-pressure pneumoperitoneum for reduction of shouldertip pain following laparoscopy. Br J Surg. 2000; 87: 1161-65.

24. Fredman B, Jedeikin R, Olsfanger D, et al. Residual pneumoperitoneum: a cause 
of postoperative pain after laparoscopic cholecystectomy. Anesth Analg. 1994; 79: 1524.

25. Gutt CN, Oniu T, Mehrabi A, et al. Circulatory and respiratory complications of carbon dioxide insufflation. Dig Surg. 2004; 21(2): 95-105.

26. Grass JA. Epidural analgesia. Probl Anesth. 1998; 10: 445.

27. Wheatley RG, Schug SA, Watson D: Safety and efficacy of postoperative epidural analgesia. $\mathrm{Br}$ J Anaesth. 2001; 87: 47.

28. Joris JL, Noirot DP, Legrand MJ, Jacquet NJ, et al. Hemodynamic changes during laparoscopic cholecystectomy. Anesth Analg. 1993; 76: 1067-71.

29. Wahba RW, Beique F, Kleiman SJ. Cardiopulmonary function and laparoscopic cholecystectomy. Can J Anaesth. 1995; 42: 5163.

30. Oedeberg S, Ljungqvist O, Svenberg T, et al. Haemodynamic effects of pneumoperitoneum and the influence of posture during anaesthesia for laparoscopic surgery. Acta Anaesthesiol Scand. 1994; 38: 276-83.

31. Thune A, Appelgren L, Haglind E. Prevention of postoperative nausea and vomiting after laparoscopic cholecystectomy. Eur J Sur. 1995; 161: 265-8.

32. Iitomi $\mathrm{T}$, Toriumi $\mathrm{S}$, Kondo $\mathrm{A}$, et al. Incidence of nausea and vomiting after cholecystectomy performed via laparotomy or laparoscopy. Masui. 1995; 44: 1627-31.

33. GautamB,ShresthaBR,LamaP,RaiS.Antiemetic prophylaxis against postoperative nausea and vomiting with ondansetron-dexamethasone combination compared to Ondansetron or Dexamethasone alone for patients undergoing laparoscopic cholecystectomy. KU Med Journ. 2008; 6:3(23): 319-28.
34. Tzeng JI, Hsing $\mathrm{CH}$, Chu CC, et al. Low-dose dexamethasone reduces nausea and vomiting after epidural morphine: a comparison of metoclopramide with saline. J Clin Anesth. 2002; 14: 19.

35. Jensen $P$, Mikkelsen $T$, Kehlet $H$. Postherniorrhaphy urinary retention: effect of local, regional and general anesthesia, a review. Reg Anesth Pain Med. 2002; 27(6): 612-17.

36. Egbert LD, Bant GE, Welch CE, et al. Reduction of postoperative pain by encouragement and instruction of patients. N Engl J Med. 1964; 207: 824-27.

37. Freund FG, Bonica JJ, Ward RJ, et al. Ventilatory reserve and level of motor block during high spinal and epidural anesthesia. Anesthesiology. 1967; 28: 834-37.

38. Bailey PL, Rhondeau S, Schafer PG, et al; Doseresponse pharmacology of intrathecal morphine in human volunteers. Anesthesiology. 1993; 79: 49.

39. Samii K, Chauvin M, Viars P. Postoperative spinal analgesia with morphine. Br J Anaesth. 1984; 53: 817-20.

40. Gwirtz KH, Young JV, Byers RS, et al; The safety and efficacy of intrathecal opioid analgesia for acute postoperative pain: Seven years' experience with 5969 surgical patients at Indiana University Hospital. Anesth Analg. 1999; 88: 599.

41. White PF, Kehlet H, Neal J M, Schricker T, et al. The Role of the Anesthesiologist in FastTrack Surgery: From Multimodal Analgesia to Perioperative Medical Care. Anesth Analg. 2007; 104: 1380-96.

42. Gaba DM, Fish KJ, Howard SK. Basic principles of crisis management in anesthesiology. Crisis management in anesthesiology; Philadelphia, Pensylvania: Churchill Livingstone Inc; 1994. p. 2. 